\title{
Téoros
}

Revue de recherche en tourisme

\section{Un bref regard sur l'architecture de la villégiature et du tourisme}

\section{Gérard Beaudet}

Volume 15, numéro 1, printemps 1996

Les Laurentides, quelles Laurentides?

URI : https://id.erudit.org/iderudit/1075053ar

DOI : https://doi.org/10.7202/1075053ar

Aller au sommaire du numéro

Éditeur(s)

Université du Québec à Montréal

ISSN

0712-8657 (imprimé)

1923-2705 (numérique)

Découvrir la revue

Citer cet article

Beaudet, G. (1996). Un bref regard sur l'architecture de la villégiature et du tourisme. Téoros, 15(1), 39-42. https://doi.org/10.7202/1075053ar d'utilisation que vous pouvez consulter en ligne.

https://apropos.erudit.org/fr/usagers/politique-dutilisation/ 


\title{
UN BREF REGARD SUR LARCHITECTURE DE LA VILLÉGIATURE ET DU TOURISME
}

\author{
Gérard Beaudet
}

\section{Gérard Beaudet est architecte et urbaniste. II enseigne à l'Institut d'urbanisme de la Faculté de l'aména gement, Université de Montréal.}

Les petites malsons de ferme de plèce-sur-plèce et les bâtuments villageols plus élaborés de l'époque victorienne ont constitué le paysage architectural de la nalssance du tourlsme et de la villéglature dans les
Une telle architecture de villégiature a été implantée en d'autres lleux desservis par chemin de fer, le long de routaouals ou de la vole du CP en direction de Mont-Laurler. Nulle part on ne découvre toutefols une telle concentration qu'à Sainte-Agathe-des-Monts. En dếplt des pressions du développement immobilier, on y conserve un certain nombre de ces résidences secondalres blotties au creux d'aménagements paysagers plus ou moins formels.

Laurentides. Très tôt cependant, les villéglateurs transposeront en ces lleux qu'lls adoptent une archiltecture distinctive qul essalme à l'ếchelle du nord-est américaln. II en sera de mème, quolque avec quelques années de retard, dans le domaine de l'hébergement hồteller.

Larchitecture vernaculaire qul caractérlse ces pays de colonisation récente n'en conservera pas molns un attrait certain dont on s'inspirera dans les années 1930 et 1940 , au moment où on construlra quelquesuns des plus importants complexes d'hébergement touristlque. Ouant à l'architecture vllageolse du tournant du slècle, elle sera progressivement redécouverte à compter des années 1980 et devlendra une référence obllgée pour de nombreux projets à vocation commerclale en secteurs touristiques.

Plusleurs autres courants architecturaux marqueront le paysage du tourlsme et de la villéglature dont, au premler plan, le modernisme des

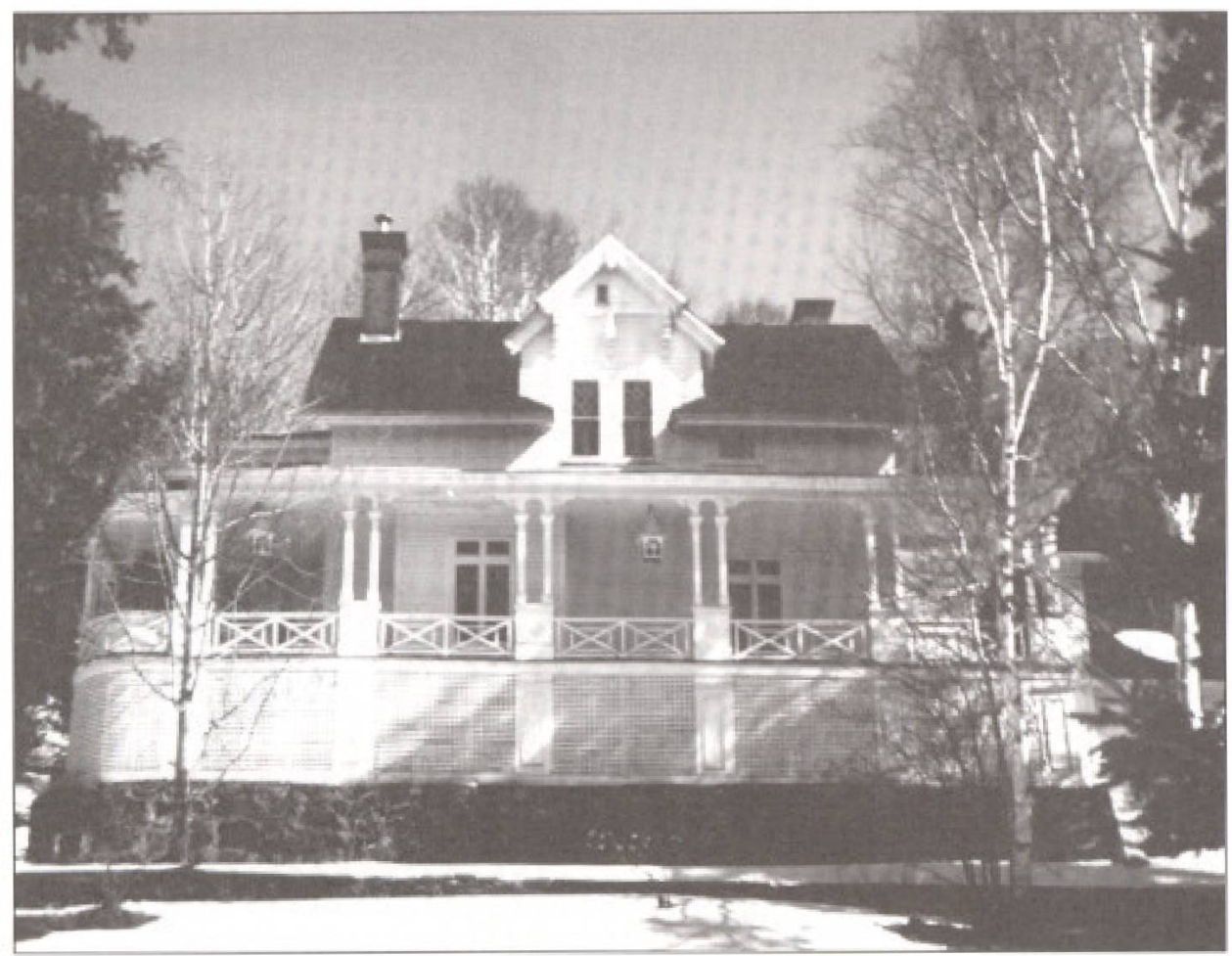
années 1950-1980. Ie postmodernisme des années 19801990 ainsl que les styles néoquébécols et alpin des annécs 1960-1970. C"est à un bref survol d'un siècle de cette architecture laurentidienne que convle cet article 1.

\section{La phase pionnière (1890-1930)}

Les résidences secondalres érigées à Sainte-Agathe-desMonts incarnent, plus que partout allleurs dans les Laurentldes, cette poussée de villégiature qul accompagne le déplolement du réseau ferrovlalre dans le dernier quart du XIXe siècle. L'élite Industrielle, financière et politique se constrult, Icl comme en d'autres lieux réputés pour leurs attralts naturels ${ }^{2}$, de vastes demeures d'une architecture éclectique de style néo-Queen Anne, néo-gothique, Regency. vernaculaire ou rustique (notamment les $\log$ houses $^{3}$ ).

Résidence secondaire au lac des Sables,

Sainte-Agathe-des-Monts.

Durant cette phase pionnlère, on assistera également à la construction de chalets plus modestes et des premlères auberges. Les chalets, dont plusleurs sont implantés sur le territoire des clubs de chasse et pêche, sont, pour la plupart, d'un style rustique caractérisé par l'utillsation du bols rond. Quant aux premlères auberges, elles s'apparentent aux hôtels de village et aux vastes maisons rurales. La partle la plus ancienne du Gray Rocks Inn et le chalet Cochand (incendié en 1920) en constitualent des exemples. II s'agissalt essentiellement d'imposants bâtiments de bols de deux ou trols étages, coiffés d'une tolture à deux ou à quatre versants et ceinturés de vastes galerles couvertes permettant de jouir du paysage. 


\section{L'époque de la grande hôtellerie (les années 1930-1940)}

Les années 1930-1940 seront celles de la grande hôtellerle. Malgré la crise, on verra se multipller les établlssements dhébergement. Le style rustique sera à l'honneur. II constituera la réponse à cet engouement pour le passé qul caractérise une époque où la modernité falt apparaître la dlstance crolssante qul s'instaure entre la société industrielle et les soclétés qui l'ont pré́cédếe.

L'amorce de l'Inventaire des oeuvres d'Arts de la province de Québec, l'étude de l'architecture anclenne à l'Université McGill, la réalisation d'enquêtes ethnologlques, l'étude du mobiller traditlonnel à l'École du meuble alnsi que la promotion d'un tourisme plus enraciné dans les tradittons locales constitueront la tolle de fond de cette production architecturale.

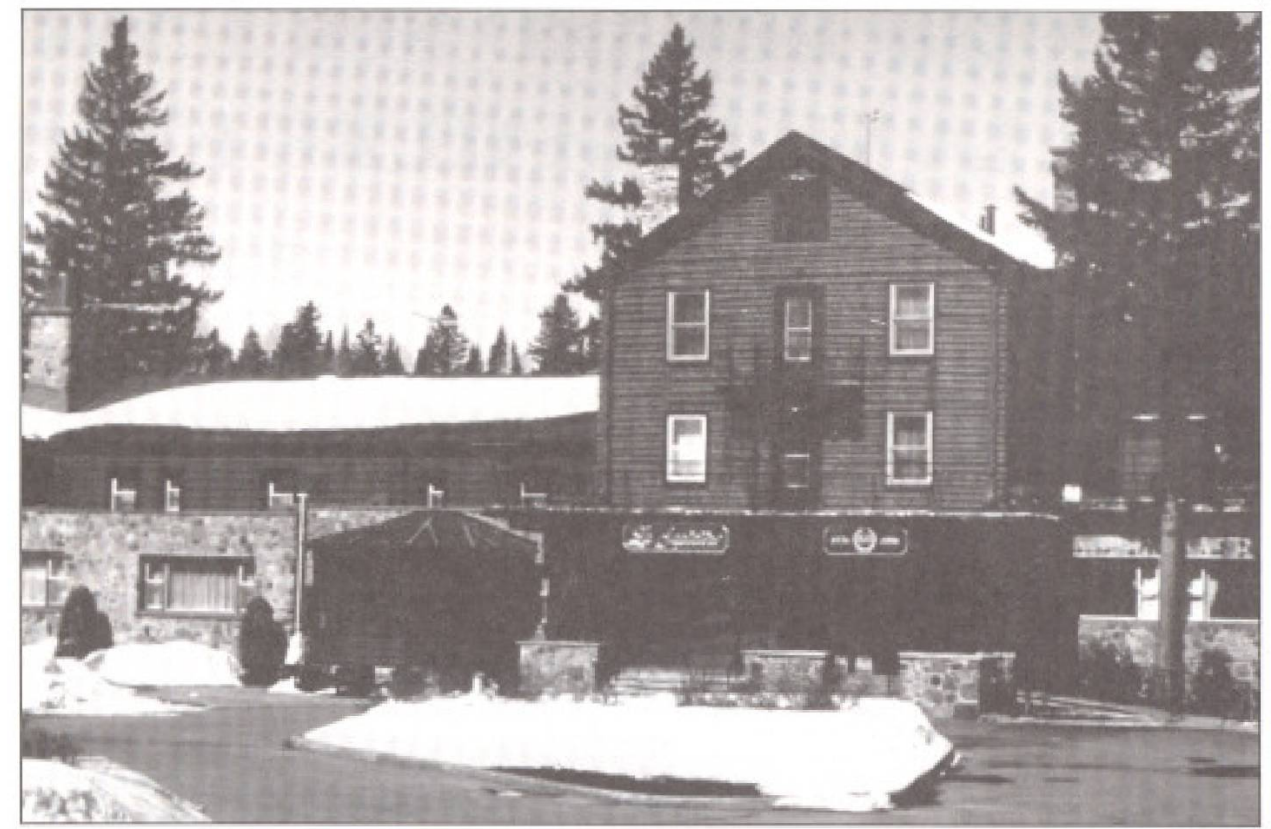

Le chalet de skl de John H. Molson, constrult au début des années 1930 à Pledmont, Illustre ce courant architectural dominant. II s"aglt d"une petite malson de plèce-sur-plèce quI reprodult l'essentiel des caractères de l'architecture vernaculaire d'un pays de colonisation ${ }^{4}$.

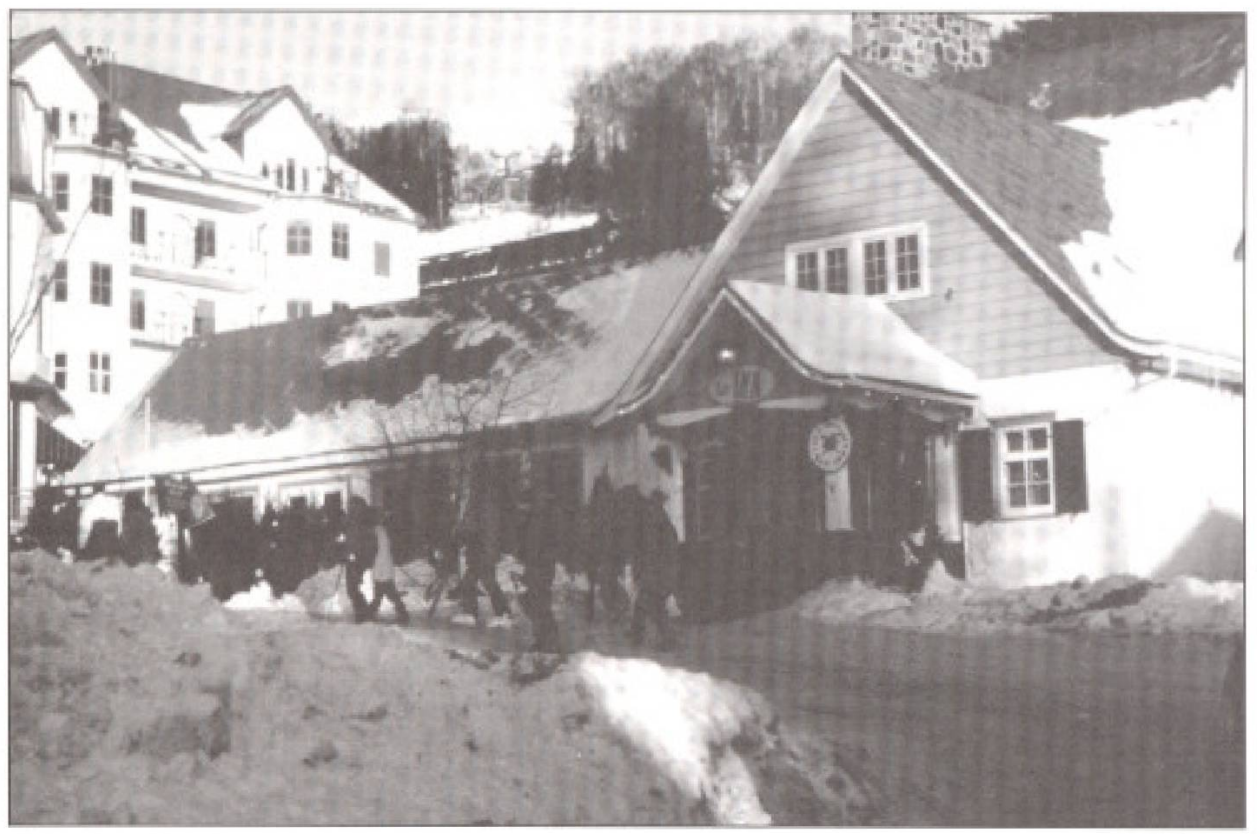

La Sapinière. Le corps central du bâtiment témoigne de l'architecture rustique des années 1930.

ment avec éloquence de ce mouvement. Mals II s'aglt, dans ces derniers cas, d'une architecture qul emprunte à une technique scandinave de construction en billes de bols. C'est donc moins l'authentucité de la référence à la tradition qui compte que l'effet obtenu. Il sera tout simplement grandlose dans le cas du Château Montebello ${ }^{5}$. le plus grand bâtIment du genre au monde.

L'auberge Le Chanteclerc pulsera davantage à la tradition architecturale locale. Le bâtiment de plerre et de plèce-sur-plèce à la toiture à la Mansard s'inspirera en effet, quolque très librement, des manoirs de la Nouvelle-France, popularlsés par les écrits de Plerre-Georges Roy. La construction, à l'entrée du site, de chalets Imitant la malson des colons ainsi que d'une chapelle reprodulsant les petites églises d'avant la Conquête confirmera cette volonté d'enracinement destiné à fonder une amblance propice à la détente et au dépaysement.

Cette référence à l'esprit des lleux sera également à l'origine du concept archltectural du Mont Tremblant

Le Mont-Tremblant Lodge et le Bourg Saint-Bernard.

Cinquante ans d'histoire, deux représentations du Québec.

Le Nymark's Lodge de Saint-Sauveur (incendlé à la fin des années 1970), I'Alpine Inn de Sainte-Marguerite-du-lacMasson, la Sapinière de Val-David et le Château Montebello (Lucerne-In-Quebec Seigntory Club house) témolgnent égale-
Lodge. Les quelque quatre-vingt-dix bátiments s'inspirant de l'archittecture vernaculalre réglonale formeront un véritable hameau dominé par le clocher de la petite chapelle, répllque fidèle d'une des églises de l'île d'Orléans. Il s'agira de l'ensemble du genre de loin le plus achevé des Laurentides ${ }^{6}$.

Si le rustique est également à l'honneur pour les premlers bâtiments du domaine de l'Estérel (l'auberge La Polnte Bleue 
el une vingtaine de chalets). on lui substituera rapidement la volumétrie blanche et lisse du style international. Les formes épurées du bătıment abritant cinéma et boutıques, de l'auberge, du pavillon des sports et de la dizaine de cottages en font un ensemble architectural exceptionnel qui pulse au répertolre de l'avant-garde européenne des années 1920. II faudra attendre l'après-guerre pour que ce style fasse une vêritable percée dans les Laurentides. Rarement atteindra-ton toutefols la qualité de cet ensemble architectural méconnu? ${ }^{7}$.

\section{L'essor de la villégiature (les années 1950, 1960 et 1970)}

Les décennles 1950,1960 et 1970 seront marquées par un développement sans précédent de la vllléglature. Les fonds des vallées et les versants des collines se couvriront de chalets et, de plus en plus, de résidences secondaires fréquentées a longueur d'année. Les courants néoquébécols et d'inspiratıon alpine domineront les deux dernlères décennles de celte pérlode. Le chalet alpin, caractérisé par son mur plgnon abondamment fenestré et ses vastes galeries abritées par le débord de la tolture, sera priviléglé dans le cas des versants. Quant à la malson dite québécolse, dont le carré de plèce ou de plerre est coiffé d"une tolture à larmlers percée de lucarnes, clle sera préférée dans les creux et aux abords des vlllages. La halte la Porte-du-Nord Incarnera également ce courant en transposant dans les BassesLaurentides une volumétrle s'inspirant de larchitecture de plerre de la vallée du Saint-Laurent.

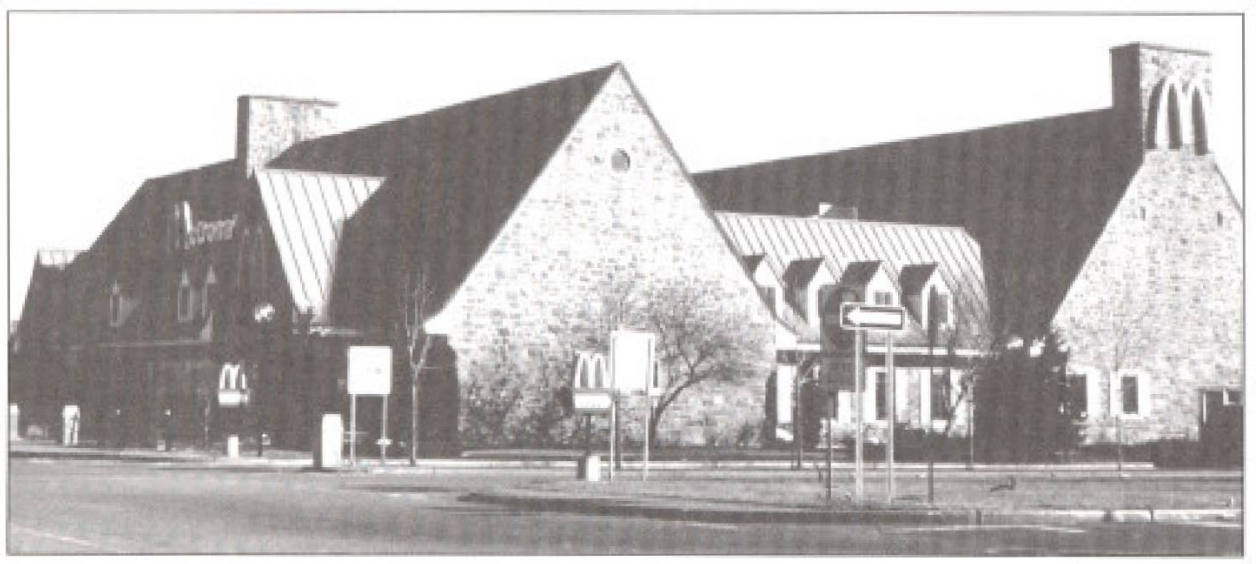

La Porte du Nord.

La référence au passé du Québec demeure une constante.

Larchitecture dite moderne ne fait, dans le domaine de la villéglature, que quelques timides percées. En revanche, elle domine dans l'hébergement. Les nombreux motels qul se multipllent, en particulier le long de la route 117 , empruntent en effet allègrement à ce style dont les principales caractéristlques résident dans les formes très épurécs, dans l'emplol de matérlaux produits en usine et dans l'absence de référence à l'histolre ou à l"architecture régionale. La production sera cependant, dans son ensemble, passablement banale et plusieurs bátiments construits durant cette période ont été abandonnés ou ont défà subl d'importantes transformations.
Cette modernité gagnera également le coeur des villages et des petites villes dont les bấtiments à vocation commerclale seront allègrement rénovés, transformés ou agrandis pour tirer partı et témolgner de la prospérité économique cependant que de nouvelles constructions stajouteront sans rapport avec leur environnement. Ce qui est aujourd'hul davantage perçu comme un manque évldent de sensibilité à l'égard du patrimoine et des paysages étalt alors sıgne de progrès. Il faut dire que la problématique patrimonlale ne retenait alors pas vérilablement l'attention dans les Laurentides. Tout y semblalt (relativement) trop récent et trop modeste.

Parmi les rares initlatives à incidence patrimonlale de la pérlode qui peuvent être signalées, on retlendra la constitution du village de Séraphin à Val-David et celle du village Canadlana à Rawdon. L'un et l'autre ensembles de bâtiments déménagés ou constrults dans un style d'époque constituent en quelque sorte des enclaves en marge de la modernité triomphante qui n'est guère remise en question, du molns dans la réglon ${ }^{8}$.

\section{Le retour en force du passé (les années 1980-1990)}

Les quinze dernlères années de la production anchitecturale dans les Laurentıdes peuvent être caractérisées par le retour en force du passé. Cela se manlfeste, entre autres, par les efforts consentls par certaines municipalités pour protéger et revaloriser le patrimolne bâtı local. C'est alnsı que Prévost (Shawbridge), Pledmont, Saint-Sauveur-des-Monts, Salnte-Agathe-des-Monts, SalnteMarguerite-du-lac-Masson. SalntDonat, Sainte-Adèle, Salnt-Jovite et Wakefleld. pour ne nommer que ces munlcipalités, ont entrepris diverses démarches en vue de mleux assurer la conservation et la mise en valeur de leur patrimoine.

La prépondérance de la justification touristique falt néanmoins parfols problème. Le patrimoine est en effet souvent le prétexte d'Interventions quil cherchent molns à le préserver qu'à constituer un cadre architectural conforme à l'Image dominante véhlculée par le tourisme de masse à l'échelle du nord-est amérlcaln. Le petit Hameau de SaintJovite, le Village des Monts à Sainte-Agathe-des-Monts et le Bourg Saint-Bernard de la station Tremblant en constituent des lllustrations particulièrement éloquentes. Malgré un voisinage d'Intérêt patrimonial, ces réalisations architecturales se suffisent à elles-mêmes et pourralent avolr été implantées un peu partout, pourvu qu'Il y eut affluence touristlque. Pre, elles devlennent souvent la référence en matière de patrimoine.

Cette prolifération d'une architecture d'imitation comporte donc certains risques. C'est ce que montre l'exemple de la vallée de Saint-Sauveur. On y a en effet été contralnt de constituer le village de Saint-Sauveur en site du patrimoine pour le protéger des conséquences d'un développement qui a 
pris nalssance à ses abords mals qui en compromettalt l'avenlr en ralson des pressions générées.

L'émergence d'une sensibilité patrimonlale n'est par ailleurs pas étrangère à la montée du courant postmoderne au cours des années 1980. Lun et l'autre s"inscrivent pour partie en réaction contre le modernlsme des trols décennles précédentes qui a prévalu dans les domaines de l'hébergement et du commerce. Mals, contralrement à l'architecture d'imitathon, le courant postmoderne, dominant dans les projets de consolidation et de modemisation des stations de skl et de l'hébergement, a produit certains batiments de grande qualIte au nombre desquels figurent le Pavillon 70 à SaintSauveur-des-Monts et le Village Mont-Tremblant.

\section{事秦}

L'architecture du tourisme et de la vllleglature dans les Laurentides constitue à la fols le support, le décor et une des matlères premlères de la vocation touristlque réglonale. Ce trop bref survol a suggéré le rlchesse et la diversité d'une production qul s'étend sur plus d'un slècle. Elle demeure toutefols Insuffisamment connue. Aussi Importe-t=II de la mleux documenter pour éviter une dllapldation de cette richesse.

\section{Bibliographie}

Bergeron, Claude, Architecture du XXe slècle au Québec, Musées de la clvilisatıon/Méridien, 1989.

Dubé. Claude. Deux cents ans de villéglature dans Charlevolx. Les Presses de I'Université Laval 1986.

Gagnon Pratte, France, Malsons de campagne des montréalals 1892-1924: l"archltecture des frères Maxwell, edltlons du Mérldien. 1987.

Garceáu, Henri-Paul (1990). Ghronlque de l'hospltallté hótellère du Québec de 1880 à 1940 . Les publications du Québec/Méridien.

Noles

1 II s'agit don survol d'autant plus partiel qu"il traile surtout des laurentides du

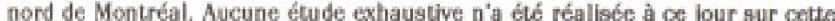
architecture du tourisme et de la villeglacure. Uné part significatwe de la production architecturale reste de ce fait méconnue. Cela est encore plus vral

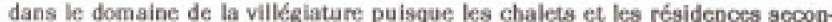
daires sont habituellement peu visibles. Seules les revues darchitecture permettent de se laire une lide de la production contemporaine.

2 Lil Malbaie, Pointe-au-Pic, Cacoung et North Hauev constutuent les hauts llew de la villégiature du tournant du siêcle au Ouébec. Ces ensembles, moins soumis à la pression urbaine ot de villéglature, ont mieux résiste que SalnteAgathe-des-Monta. Pour une description de la villégiature dans Charlewoix, voir Fointage de Claude Dubé (1986).

3 Suc les log houses de Sainte-Agathe-des-Monts, voir le chapitre que leur congicke l'ourrage de France Gagnon Praue (1987).

4 Sur celte période, voir Claude Bergeron (1989) p.126 at sulvantes.

5 Idem. p 134 et 135

18 Le scherna daménagement de la MRC at le plan drurbanitume local reonnabo sent l'intérét patrimonial de cet ensemble. Sa conservation s'imposait dond dans le cadre du projet de developpenent de la station Tremblant.

7 La bròse histoln du domaine Batérel explique en nartie que ce complexe archltectural soit si peu connu. Quelques annés apres l'ouverture de l'́tablisse ment les biens du proprielaire le baron Empain, sont mis sous sequegtre par le pouvernement canadien et Thotel est converti en mess pour les offlelers de l'Aviation canadienne. Un incandie détruira par ailleurs une partie du bátiment abritant salle de cinema, boutiques et tcuries. II faudra attendre la fin des anntes 1950 pour que l'ancien pavillon des sports, Érigd sur le bord du late Dupuis, soit. interged dans l'hotel Esterel. Un CLSC et l'administration muaict. pale logent acuellement dans l'ancien cantre culturel et commercial, l'bótel, passablement bien conservé, a duc conwerti an centre de convalescence cepen dant que les petita cottages ont été plus ou moins transformés par leurs propriélaires. II importerait qu'un inventaire architectural de cet ensemble de bâtiments soit fait et que deg travaux de conservation sojent rélisés.

8 Ces ritalisationg s'inscriwent dans la llenée du Upper Canada village et du Village québeticois d'Mntan de Drummondville. La village Canadiana avalit couteFols sud constitud d'abord comme une collaction prives. Ce g'eqt que graduellement nue siest forgbe lidede douvrir th site et ses collections de batiments. d'ouvrages et d'objets aux visiteurs. Ouant au Village de Seraphin. Ils faic épalement partio de ces lieux néa de la diffugion de téléromang (Le temps d'une Paix, Les Filleg de Caleby. Bien que ong diwereg réalientiong puisgent contribuer à la saumegarde du patrimoine. il ne gagit pas à proprement parler de conservation et de mise en valeur au gens où on l'cnlend habituellement. 